
\title{
28 Research Suare \\ For a systematic comprehension of GAB2: an analysis based on pan-cancer
}

\section{Yi Yin}

Nantong University

\section{Zongyu Guan}

Nantong University

\section{Zhaoyang Ou}

Nantong University

\section{Min Tang}

Nantong University

\section{Yong Li}

Nantong Tumor Hospital(The Affiliated Tumor Hospital of Nantong University)

\section{Can Zhang}

Nantong Tumor Hospital(The Affiliated Tumor Hospital of Nantong University)

\section{Li Zhang}

Nantong Tumor Hospital(The Affiliated Tumor Hospital of Nantong University)

Aiqin He ( $D$ haq5300447@ntu.edu.cn )

Nantong Tumor Hospital(The Affiliated Tumor Hospital of Nantong University)

\section{Research Article}

Keywords: GAB2, cancer, prognosis, phosphorylation, Immune infiltration

Posted Date: January 27th, 2022

DOI: https://doi.org/10.21203/rs.3.rs-1224365/v1

License: (c) (i) This work is licensed under a Creative Commons Attribution 4.0 International License. Read Full License 


\section{Abstract}

Backgroud: The potential function of Grb2-associated binder2(GAB2) in human ErB-2 (Her-2) overexpressed breast cancer cells has been reported. GAB2 may be a potential target for clinical treatment of Her-2-overexpressed breast cancer. However, whether GAB2 plays a pivotal role in other cancers as well is unclear. Here, we aimed to explore the role of GAB2 in pan-cancer.

Methods: We explored the role of GAB2 in tumor prognosis and tumor immunity in a wide range of cancers through a variety of databases. The expression and prognostic correlation of GAB2 in 33 tumors were explored using The Cancer Genome Atlas Project(TCGA), The Genotype-Tissue Expression(GTEx) and The Clinical Proteomic Tumor Analysis Consortium(CPTAC) databases. We also described the expression of phosphorylated GAB2 meanwhile analyzed the correlation between GAB2 and tumor immunity in pan-cancer.

Results:The expression level of GAB2 is different between normal and tumor tissues in pan-cancer, and phosphorylated GAB2 also plays an important role in tumor development. The expression level of GAB2 was also statistically significant with the Overall Survival(OS) of tumor patients. There was a positive correlation between GAB2 and CD8+T immune infiltration in pan-cancer.

Conclusion: The expression levels of both GAB2 and phosphorylated GAB2 were different in pan-cancer, and there was a statistical significance between the expression level of GAB2 and tumor prognosis. In addition, GAB2 may be a potential target for tumor therapy due to its positive correlation with immune infiltration.

\section{Introduction}

Cancer is the first or second leading cause of death in many countries(1). At present, the main treatment for cancer is still surgery, chemotherapy and radiotherapy.

In recent years, immunotherapy has gradually become the key treatment for cancer(2). However, the vast majority of people will eventually develop resistance to chemotherapy(3). Therefore, it is very necessary to investigate the potential therapeutic targets of breast cancer.

GAB2 is a major member of the Gab family proteins, which contains GAB1, GAB2, GAB3, GAB4 Drosophila daughter of sevenless(DOS), and Caenorhabditis elegans suppressor of Clr-1(COS)(45).GAB2 is mainly composed of a pleckstrin homology $(\mathrm{PH})$ domain at the N-terminus, proline-rich motifs, and multiple tyrosine residues(6-7).PH domain can identify membrane component, especially phosphatidylinositol 3,4,5-triphosphate (PIP3), contributing to membrane recruitment. Proline-rich motifs contains PXXP domain structure, binding to the proteins which include $\mathrm{SH} 3$ domain. multiple tyrosine residues can combine with with $\mathrm{SH} 2$ domain structure of the protein(8). The previous study has reported that GAB2 plays an important role in the metastasis of breast cancer(9).Davis et al demonstrated that the expression level of GAB2 is associated with the prognosis of ovarian cancer(10). 
However, the relationship between the expression level of GAB2 and other tumors in the TCGA database remains unclear. Therefore, we used bioinformatics to explore the relationship between the expression level of GAB2 and other tumors.

\section{Methods}

Gene expression analysis

We logged into oncomine and analyzed the expression of GAB2 mRNA levels in different tumors. We input GAB2 in Gene_DE module of Timer2.0 website to obtain the expression of GAB2 in normal and tumor tissues of different tumors in TCGA project. We combined the TCGA and GTEX databases in GEPIA to further analyze the difference in the expression level of GAB2 in these tumors between normal and tumor tissue. P-value cutoff $=0.01$, log2FC (fold change) cutoff $=1$.

Survival prognosis analysis

Kaplan-Meier Plotter online tool was used to analyze the relationship between GAB2 expression and OS in pan-cancer. We analyzed the relationship of GAB2 expression with OS in BLCA(Bladder Carcinoma), BRCA(Breast invasive carcinoma), KIRC(Kidney renal clear cell carcioma), LUAD(Lung adenocarcinoma), LUSC(Lung squamous cell carcinoma), READ(Rectum adenocarcinoma), SARC(Sarcoma), THYM(Thymoma), THCA(Thyroid carcinoma) and UCES(Uterine Corpus Endometrial Carcinoma).

Phosphorylation expression analysis

The expression level of GAB2 phosphorylation was explored by using the ualcan portal, an interactive web resource for analyzing cancer omics data. We used CPTAC database to analyze the expression of GAB2 phosphorylation in normal and tumor tissues in LUAD(Lung adenocarcinoma), BRCA(Breast invasive carcinoma), UCEC(Uterine Corpus Endometrial carcinoma), OV(Ovarian cancer), COAD(Colon adenocarcinoma) and KIRC(Kidney renal clear cell carcinoma).

Correlation and enrichment analyses

The association between GAB2 and other genes was analyzed by the ovarian cancer data in the TCGA database,and further predict the function of GAB2, including related pathways. Finally, we selected the first 300 genes that were positively correlated with GAB2 for analysis. Gene Ontology (GO) analysis was performed using EnrichGO function in the R package "clusterProfiler".

Immune cell infiltration

We selected the Immune module in the Timer 2.0 online tool, then input GAB2 in Gene Expression, Immune Infiltrates selected CD8+T cells, and finally submitted. The T cellCD8+ EPIC mode was chose.

\section{Results}


In order to explore the expression level of GAB2 in pan-cancer, we detected the mRNA expression level of GAB2 using Oncomine database. As shown in Figure 1A, the expression level of GAB2 in gastric cancer, leukemia and lymphoma was significantly increased, and the expression level also was higher in breast cancer and head and neck cancer. Meanwhile, the expression of GAB2 was lower in cancers, including esophageal cancer, head and neck cancer, leukemia and lung cancer.

In addition, we further analyzed the expression of GAB2 in TCGA by using Timer software, and the results were shown in Figure 1B. GAB2 expression was significantly lower in BLCA, BRCA, CESC(Cervical and endocervical cancer), COAD, HNSC(Head and Neck squamous cell carcinoma), LUAD, LUSC, PRAD (Prostate adenocarcinoma), READ (Rectum adenocarcinoma), UCEC than that in normal tissue. However, we also found that the expression level of GAB2 was higher in $\mathrm{CHOL}$ (Cholangiocarcinoma), $\mathrm{KICH}($ Kidney Chromopphobe), KIRC, LIHC(Liver hepatocellular carcinoma), PCPG(Pheochromocytoma and Paraganglioma), THCA than that in normal tissue.

Because some tumors in the TCGA data lacked normal tissue, we combined the TCGA and GTEx databases in GEPIA to analyze the difference in the expression of GAB2 in normal tissue and tumor tissue. The results were shown in Figure 1C. The expression levels of GAB2 were different between normal tissues and tumor tissues in DLBC(Lymphoid Neoplasm Diffuse Large B-cell Lymphoma), LGG(Brain Lower Grade Glioma) and UCS(Uterine Carcinosarcoma).

The relationship between the expression level of GAB2 and prognosis in cancer

We further explored the relationship between GAB2 expression levels and survival in pan-cancer patients. Kaplan-Meier Plotter tools were used to analyze the relationship between GAB2 mRNA levels and survival of patients with hematoma. We found that the Overall Survival(OS) of patients with high level of GAB2 mRNA was shorter than that with low level of GAB2 mRNA in BLUC, LUSC and UCEC. At the same time, we still found the opposite phenomenon, tumor patients with high GAB2 mRNA level had significantly better OS than tumor patients with low levels in BRCA, KIRC, LUAD, READ, SARC(Sarcoma), THCA and THYM. (Figure 2).

Phosphorylation analysis of GAB2 in pan-cancer

We analyzed the differences in phosphorylation levels at different sites of GAB2 in normal and tumor tissues from different tumors. As shown in Figure 3, Ualcan tool was used to analyze 6 types of tumor(breast cancer, LUAD, ovarian cancer, UCEC, COAD and KIRC). In breast cancer, the phosphorylation levels of S185, T508 and Y373 sites were different between normal and tumor tissues, and phosphorylation levels in normal tissues were higher than those in tumor tissues. A similar phenomenon was observed, phosphorylation levels is significantly higher in normal tissues at S330, S505, and T353 rather than S185, T508, and Y373 in Lung adenocarcinoma. However, in ovarian cancer, phosphorylation levels at S172 and T353 sites in tumor tissues were significantly higher than those in normal tissues. 
Meanwhile, the phosphorylation level of T353 was also different in the tumor and normal tissues of colon cancer. However, in renal clear cell carcinoma, the phosphorylation level of GAB2 was not statistically significant between tumor and normal tissues. We found that the phosphorylation level at T353 differed in lung adenocarcinoma, ovarian cancer, and colon cancer. Therefore, phosphorylated T353 may be a potential target for tumor therapy.

Correlation and enrichment analyses

In order to explore the function of GAB2, we chose to use the TCGA database to analyze the correlation between GAB2 and other genes in ovarian cancer. Finally, we selected the first 300 genes related to GAB2 for enrichment analysis. As shown in Figure 4A, the top 50 genes positively and negatively correlated with GAB2 were respectively displayed. Afterwards, Functional enrichment and Gene Ontology (GO) analysis revealed that GAB2 is involved in cell movement, including integrator complex; myofibril; contractile fiber part; region of cytosol; apical junction complex; clathrin-coated pit; postsynaptic cytosol; cortical actin cytoskeleton; lamellipodium;cell-substrate adherens junction and so on(Figure 4B). The main molecular functions, biological processes and cell composition involved in GAB2 are shown in Table 1.

Correlation analysis between GAB2 expression and infiltrating immune cells

Tumor infiltrating immune cells, which participate in the composition of tumor microenvironment, affect the survival of patients with various tumors. Therefore, taking CD8+T as an example, TIMER tool was applied to explore the correlation between the expression level of GAB2 and infiltrating immune cell in pan-cancer. The result is shown in Figure 5. The expression level of GAB2 was positively correlated with tumor cell purity in PCPG and SARC, but negatively correlated with tumor cell purity in PRAD. In addition, we found that GAB2 expression was significantly correlated with CD8+T cell infiltration levels in PCPG, PRAD and SARC.

\section{Discussion}

As an intermediary molecule, GABs scaffold protein plays an important role in the physiological processes of cell proliferation, survival, differentiation, apoptosis and migration in cancer. Chenbo Ding et al. demonstrated that GAB2 induces EMT through the MEK/ERK/MMP signaling pathway, thus promotes metastasis of colon cance(11). Qian P et al. found that the increased expression of GAB2 enhanced the metastasis and invasion of breast cancer cells(12). In our study,as shown in Figure 1, GAB2 is differentially expressed in many tumors. Although the expression of GAB2 is differentially expressed in normal and tumor tissues of many tumors, the specific mechanism by which GAB2 plays a role in tumors remains unclear.

All members of the Gab family contain an $\mathrm{N}$-terminal $\mathrm{pH}$ domain with a central proline domain and multiple tyrosine residues. Phosphorylated tyrosine residues can bind to proteins containing the $\mathrm{SH} 2$ domain, such as SHP2, the Crk adaptor protein, phospholipase C (PLC), and The regulatory subunit of PI3K, p85(13). Studies have reported that the molecular structure of GAB2 contains two SHP2 binding 
sites, namely tyrosine residues $\mathrm{Y} 614$ and $\mathrm{Y} 643$. Phosphorylated tyrosine residues $\mathrm{Y} 614$ and $\mathrm{Y} 643$ bind to the SH2 domain of SHP2 to activate SHP2, thereby activating the downstream RAS/ERK (GAB2-SHP2ERK) signaling pathway(14). Bentires-Alj $M$ et al. found that in breast cancer, after co-transfection of GAB2 and ERBB2 into normal breast epithelial cells, GAB2 could lead to canceration and invasive growth of normal breast epithelial cells through the SHP2/ERK signaling pathway(15). Phosphorylation of Y452,Y476, and Y584 can bind to the $\mathrm{p} 85$ subunit of PI3K. The activation of PI3K can lead to the production of a large amount of PIPs. However, PIPs can also induce GAB2 membrane recruitment through the $\mathrm{PH}$ domain of GAB2, thus activating the downstream Akt signaling pathway(14). In the further study of chronic myelogenous leukemia(CML) found that BCR-ABL protein contained tyrosine autophosphorylation of residue Y177 can recruit GRB2-GAB2 complex, then activated including RAS/RAF/MEK/ERK, PI3K/AKT, and JAK/STAT and other signaling pathways that promote the formation of leukemia(16). In summary, phosphorylation of GAB2 plays an important role in the development of tumors through RAS /ERK, PI3K/ AKT and other signaling pathways. In this study, we also found that the phosphorylation levels at diverse sites of GAB2 were different in normal and tumor tissues. However, the exact mechanism of its action in various tumors is still unclear.

When tumor cell fragments are internalized and processed by antigen-presenting cells(APCs), especially dendritic cells, and appear on the extracellular surface of APC in combination with major histocompatibility complexes of type I and type II(MHC), the immune system generates an anti-tumor response(17). APCs interact with naive $T$ lymphocytes and trigger the activation and proliferation of tumor-specific CD4+ helper molecules and CD8+ cytotoxic T cells colonization(18). After the activation of T cells, the cytotoxic T lymplastin antigen-4(CTLA-4) was expressed on the surface of T cells. CTL A-4 binds to CD80/CD86 with high affinity and gives inhibitory signals that limit further activation of $T$ cells. This mechanism helps to maintain tolerance to host antigens on the surface of normal cells and may prevent lymphoproliferative diseases. However, tumors can escape the immune system by inducing tolerance or by developing resistance to T-cell-mediated destruction(19-21). So far, immunotherapy has been used to treat many cancers. Clinical studies have shown that vaccine therapy targeting tumor cells themselves or their abnormal expression of tumor markers is promising as immunotherapy for non-small cell lung cancer(NSCLC). Non-antigenic immunotherapies, such as anti-cytotoxic T lymphocyte antigen-4 monoclonal antibodies, talactoferin alfa, and toll-like receptor 9 antagonists, act on the activated immune system and are independent of tumor antigens and may be used for the treatment of advanced NSCLC(22). A recent clinical study of the efficacy of immunotherapy for pancreatic cancer suggests that immunotherapy alone may not be successful, but combination therapies are highly likely to be successful in the short term(23). GAB2 can regulate the PI3K/mTOR signaling pathway through the $\mathrm{pH}$ domain, and the role of the $\mathrm{PI} 3 \mathrm{~K} / \mathrm{mTOR}$ pathway in the regulation of macrophage and $\mathrm{T}$ cell activation has been reported(24-28). It has been reported that the development of GAB2-induced colitis in mice is associated with increased T cell invasion(29). In our study, we found that GAB2 expression was positively correlated with CD8T cell infiltration levels in PCPG, PRAD and SARC. Therefore, GAB2 is expected to be a potential target for tumor immunotherapy. 
In summary, we used the database to explore the correlation between GAB2 and prognosis and immune invasion in pan-cancer. The results showed that the expression level of GAB2 was different between normal and tumor tissues, and the expression level of GAB2 was significantly correlated with the prognosis of tumor. In addition, we also found that GAB2 phosphorylation also plays an important role in the development of tumors. Finally, the correlation between GAB2 and immune infiltration was analyzed, and the results demonstrated that. Based on the above results, we speculate that GAB2 is expected to be a potential target for tumor therapy.

\section{Declarations}

\section{DATA AVAILABILITY STATEMENT}

Publicly available datasets were analyzed in this study. This data can be found here: http://timer.cistrome.org/, https://www.oncomine.org/resource/login.html, http://gepia.cancerpku.cn/detail.php?gene=HCK, http://kmplot.com/private/, http://ualcan.path.uab.edu/, http://geneontology.org/, https://cistrome.shinyapps.io/timer/.

\section{AUTHOR CONTRIBUTIONS}

$L Z, Y Y$ and $A Q H$ designed this study. $Z Y O, M T, Y L$ and $C Z$ extracted the information from the databases. $Y Y$ and $Z Y G$ analyzed the data. $L Z$ and $A Q H$ supervised the entire study. $Y Y$ wrote the manuscript. All authors revised the manuscript.

COMPETING INTERESTS

The authors declare no competing interests.

\section{References}

1. F. Bray, et al., Global cancer statistics 2018: GLOBOCAN estimates of incidence and mortality worldwide for 36 cancers in 185 countries, CA: Cancer J. Clin. 2018,68

2. D.M. Pardoll, The blockade of immune checkpoints in cancer immunotherapy, Nat.Rev. Cancer 12 (2012) 252-264

3. Zheng, FM; Long, ZJ; Hou, ZJ; et al.A novel small molecule aurora kinase inhibitor attenuates breast tumor-initiating cells and overcomes drug resistance.[J].Mol Cancer Ther.2014,13(8):1991-2003

4. Ding, C; Luo, J; Fan, X; et al.Elevated Gab2 induces tumor growth and angiogenesis in colorectal cancer through upregulating VEGF levels.[J].J Exp Clin Cancer Res.2017,36(1):56

5. Ding, CB; Yu, WN; Feng, JH; et al.Structure and function of Gab2 and its role in cancer (Review). [J].Mol Med Rep.2015,12(3):4007-4014

6. W?hrle, FU; Daly, RJ; Brummer, T; Function, regulation and pathological roles of the Gab/DOS docking proteins.[J].Cell Commun Signal.2009,7():22 
7. Ke, Y; Wu, D; Princen, F; et al.Role of Gab2 in mammary tumorigenesis and metastasis. [J].Oncogene.2007,26(34):4951-60

8. Liu, Y; Rohrschneider, LR; The gift of Gab.[J].FEBS Lett.2002,515(1-3):1-7

9. Ding, C; Luo, J; Li, L; et al.Gab2 facilitates epithelial-to-mesenchymal transition via the MEK/ERK/MMP signaling in colorectal cancer.[J].J Exp Clin Cancer Res.:5

10. Davis, SJ; Sheppard, KE; Anglesio, MS; et al.Enhanced GAB2 Expression Is Associated with Improved Survival in High-Grade Serous Ovarian Cancer and Sensitivity to PI3K Inhibition.[J].Mol Cancer Ther.2015,14(6):1495-503

11. Ding, C; Luo, J; Li, L; et al.Gab2 facilitates epithelial-to-mesenchymal transition via the MEK/ERK/MMP signaling in colorectal cancer.[J].J Exp Clin Cancer Res.:5

12. Qian, $P$; Zuo, Z; Wu, Z; et al.Pivotal role of reduced let-7g expression in breast cancer invasion and metastasis.[J].Cancer Res.2011,71(20):6463-74

13. Nishida, $\mathrm{K}$; Hirano, $\mathrm{T}$; The role of Gab family scaffolding adapter proteins in the signal transduction of cytokine and growth factor receptors.[J].Cancer Sci.2003,94(12):1029-33

14. Gu, H; Pratt, JC; Burakoff, SJ; et al.Cloning of p97/Gab2, the major SHP2-binding protein in hematopoietic cells, reveals a novel pathway for cytokine-induced gene activation.[J].Mol Cell.1998,2(6):729-40

15. Bentires-Alj, M; Gil, SG; Chan, R; et al.A role for the scaffolding adapter GAB2 in breast cancer.[J].Nat Med.2006,12(1):114-21

16. Sattler, M; Mohi, MG; Pride, YB; et al.Critical role for Gab2 in transformation by BCR/ABL.[J].Cancer Cell.2002,1(5):479-92

17. Leduc, C; Quoix, E; [Vaccines for the treatment of non-small cell lung cancer].[J].Rev Mal Respir.2019,36(3):415-425

18. Kelly, RJ; Gulley, JL; Giaccone, G; Targeting the immune system in non-small-cell lung cancer: bridging the gap between promising concept and therapeutic reality.[J].Clin Lung Cancer.2010,11(4):228-37

19. Fong, L; Small, EJ; Anti-cytotoxic T-lymphocyte antigen-4 antibody: the first in an emerging class of immunomodulatory antibodies for cancer treatment.[J].J Clin Oncol.2008,26(32):5275-83

20. Tarhini, AA; Iqbal, F; CTLA-4 blockade: therapeutic potential in cancer treatments.[J].Onco Targets Ther.2010,3():15-25

21. Drake, CG; Jaffee, E; Pardoll, DM; Mechanisms of immune evasion by tumors.[J].Adv Immunol.2006,900:51-81

22. Shepherd, FA; Douillard, JY; Blumenschein, GR; Immunotherapy for non-small cell lung cancer: novel approaches to improve patient outcome.[J].J Thorac Oncol.2011,6(10):1763-73

23. Morrison, AH; Byrne, $\mathrm{KT}$; Vonderheide, $\mathrm{RH}$; Immunotherapy and Prevention of Pancreatic Cancer. [J].Trends Cancer.2018,4(6):418-428 
24. Uno, JK; Rao, KN; Matsuoka, $\mathrm{K}$; et al.Altered macrophage function contributes to colitis in mice defective in the phosphoinositide-3 kinase subunit p1108.[J].Gastroenterology.:1642-53, 1653.e1-6

25. Festuccia, WT; Pouliot, P; Bakan, I; et al.Myeloid-specific Rictor deletion induces M1 macrophage polarization and potentiates in vivo pro-inflammatory response to lipopolysaccharide.[J].PLoS One.2014,9(4):e95432

26. Steinbach, EC; Kobayashi, T; Russo, SM; et al.Innate PI3K p1108 regulates Th1/Th17 development and microbiota-dependent colitis.[J].J Immunol.2014,192(8):3958-68

27. $\mathrm{Li}, \mathrm{X}$; Wang, $\mathrm{D}$; Chen, $\mathrm{Z}$; et al.Gai1 and Gai3 regulate macrophage polarization by forming a complex containing CD14 and Gab1.[J].Proc Natl Acad Sci U S A.2015,112(15):4731-6

28. Kaneda, MM; Messer, KS; Ralainirina, N; et al.PI3KY is a molecular switch that controls immune suppression.[J].Nature.2016,539(7629):437-442

29. Wang, Z; Vaughan, TY; Zhu, W; et al.Gab2 and Gab3 Redundantly Suppress Colitis by Modulating Macrophage and CD8+ T-Cell Activation.[J].Front Immunol.2019,10():486

\section{Tables}

Table I The name corresponding to the Go entry 
G0:1902895 positive regulation of pri-miRNA transcription by RNA polymerase II

GO:0010507 negative regulation of autophagy

G0:0043087 regulation of GTPase activity

G0:0048193 Golgi vesicle transport

G0:1901096 regulation of autophagosome maturation

GO:0061726 mitochondrion disassembly

G0:0000422 autophagy of mitochondrion

G0:0007015 actin filament organization

G0:0007016 cytoskeletal anchoring at plasma membrane

GO:0051651 maintenance of location in cell

GO:0034329 cell junction assembly

G0:0010506 regulation of autophagy

Go:0045103 intermediate filament-based process

G0:0045104 intermediate filament cytoskeleton organization

G0:0061919 process utilizing autophagic mechanism

G0:0006914 autophagy

G0:0034330 cell junction organization

G0:0031532 actin cytoskeleton reorganization

G0:0007163 establishment or maintenance of cell polarity

G0:0030010 establishment of cell polarity

G0:0032039 integrator complex

GO:0030016 myofibril

G0:0044449 contractile fiber part

G0:0099522 region of cytosol

G0:0043296 apical junction complex

GO:0005905 clathrin-coated pit

GO:0099524 postsynaptic cytosol

G0:0030864 cortical actin cytoskeleton 


\begin{tabular}{ll}
\hline GO:0030027 & lamellipodium \\
\hline GO:0005924 & cell-substrate adherens junction \\
\hline GO:0005925 & focal adhesion \\
\hline GO:0043034 & costamere \\
\hline GO:0030055 & cell-substrate junction \\
\hline GO:0005913 & cell-cell adherens junction \\
\hline GO:0030863 & cortical cytoskeleton \\
\hline GO:0044448 & cell cortex part \\
\hline GO:0001726 & ruffle \\
\hline GO:0031252 & cell leading edge \\
\hline GO:0005911 & cell-cell junction \\
\hline GO:0005938 & cell cortex \\
\hline GO:0051015 & actin filament binding \\
\hline GO:0019215 & intermediate filament binding \\
\hline GO:0003779 & actin binding \\
\hline GO:0045296 & cadherin binding \\
\hline
\end{tabular}

\section{Figures}



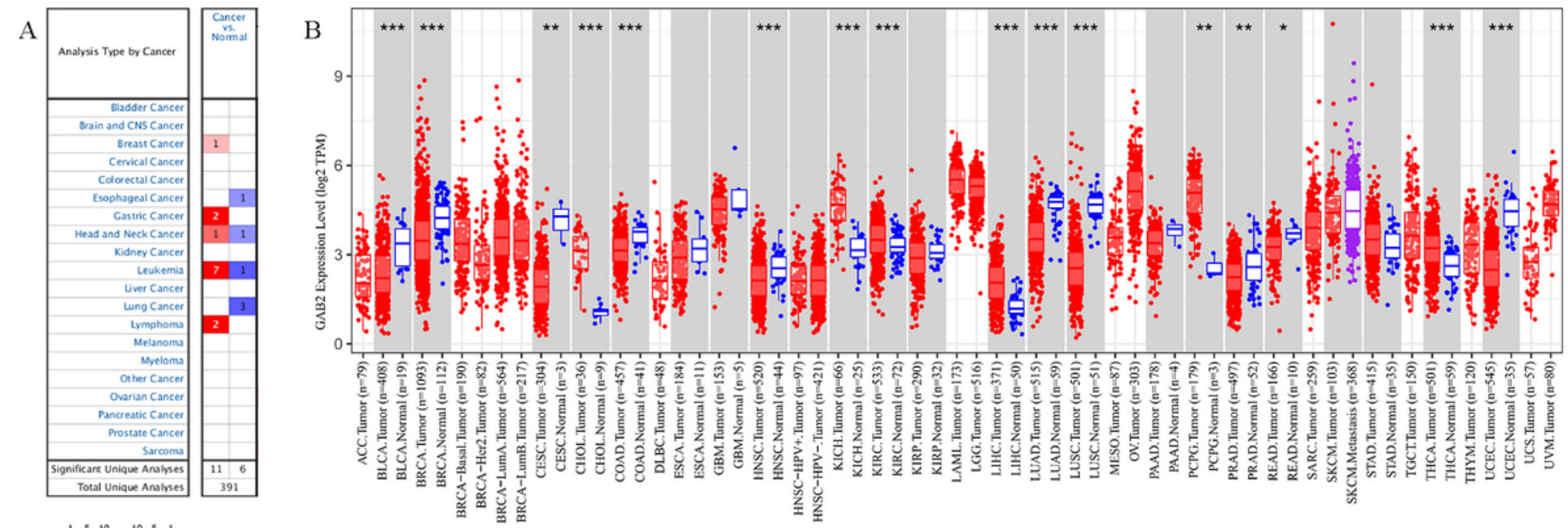

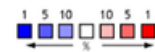

C
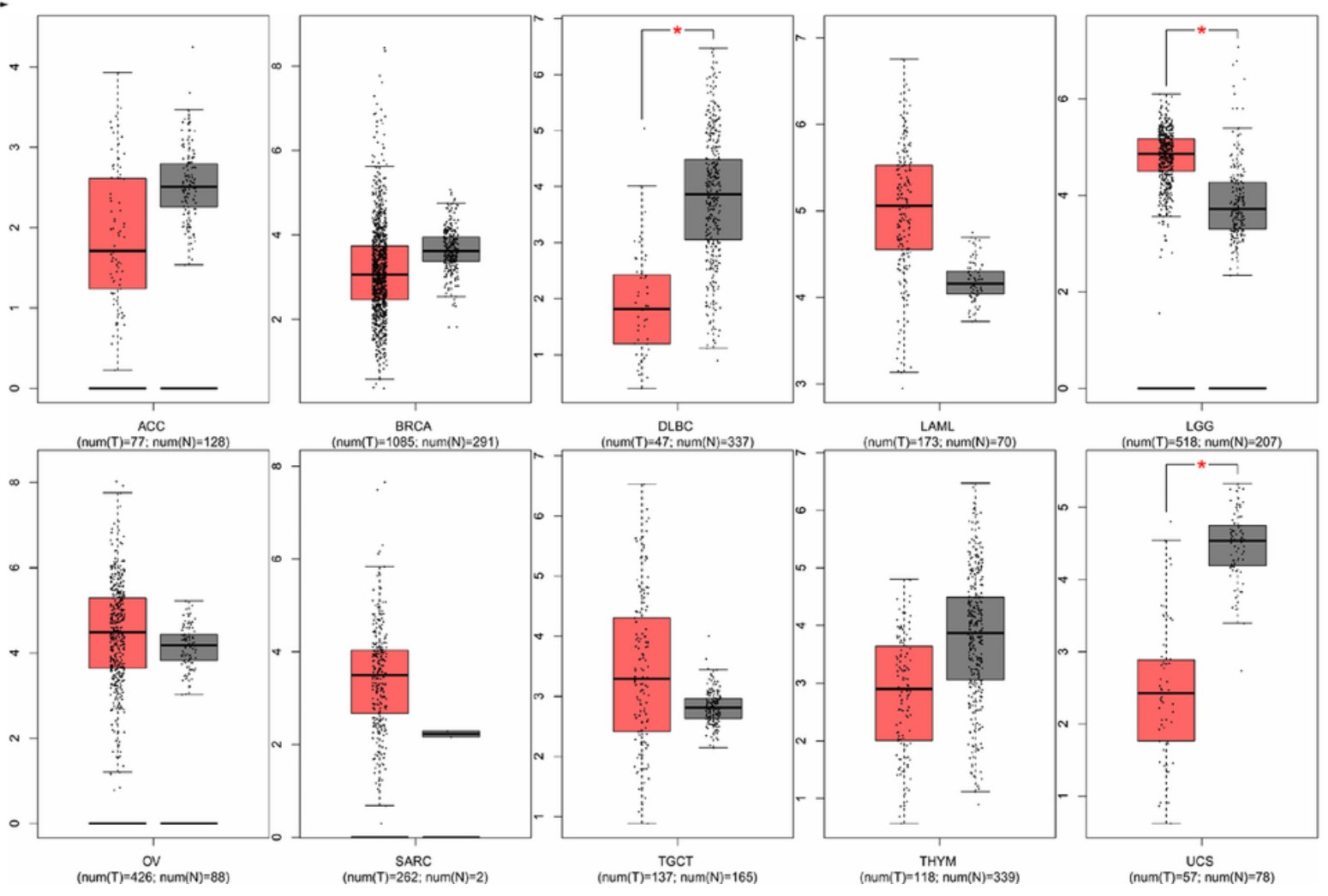

Figure 1

GAB2 expression levels in cancers. (A) Increased or decreased expression of GAB2 in different cancer tissues, compared with normal tissues in ONCOMINE. (B) GAB2 expression levels in different cancer types from TCGA data in TIMER. C GAB2 expression levels in different cancer types from TCGA and GTEx data in GEPIA Black-Normal Red-Tumor . ${ }^{*} P<0.05, * \star P<0.01, * \star * P<0.001$. 

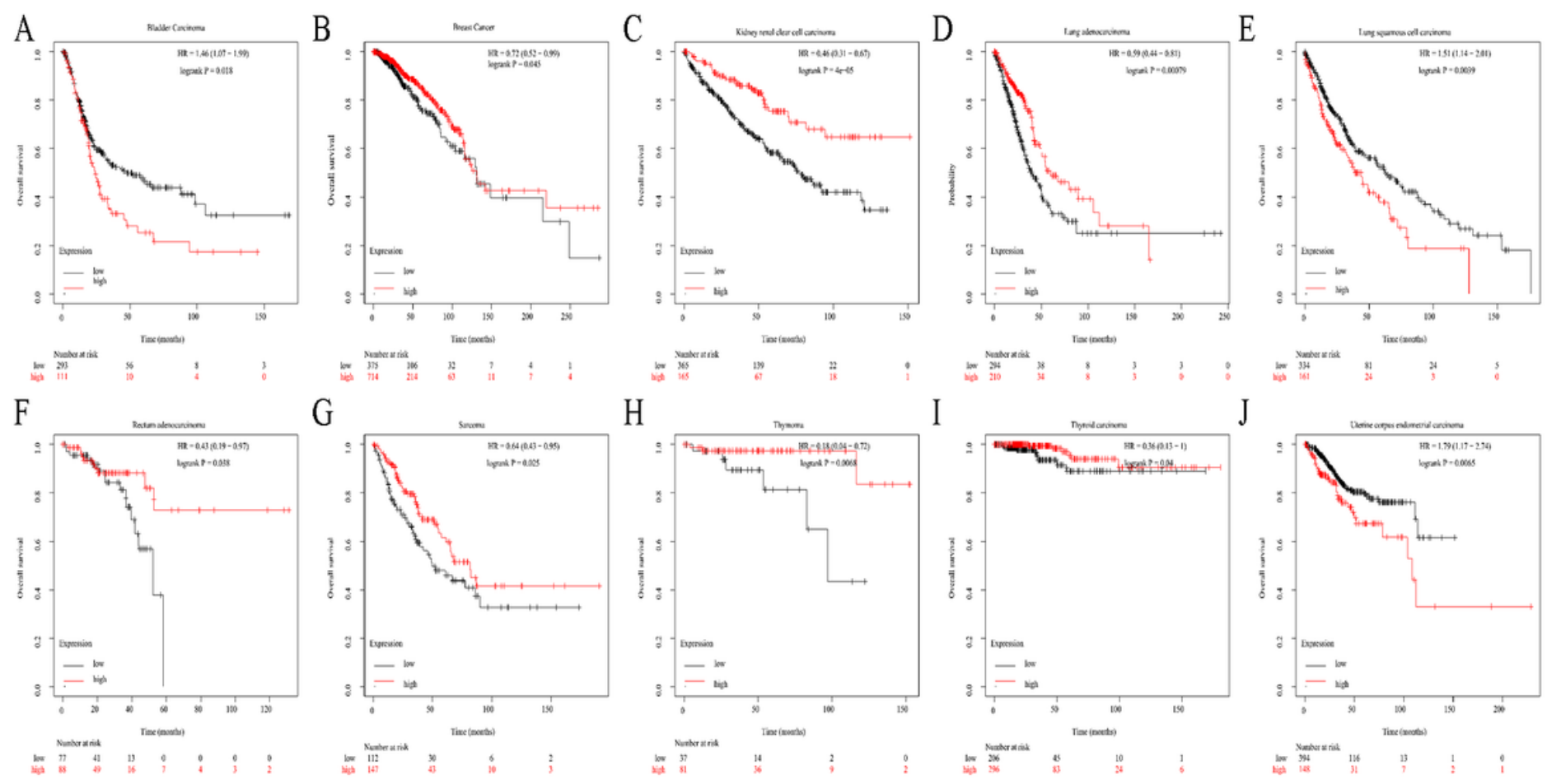

Figure 2

Kaplan-Meier survival curves comparing the high and low expression of GAB2 in different types of cancer in Kaplan-Meier Plotter. OS of (A)Bladder Carcinoma, (B)Breast Cancer, (C)Kidney renal clear cell carcinoma, (D)Lung adenocarcinoma, (E)Lung squamous cell carcinoma, $(F)$ Rectum adenocarcinoma, (G)Sarcoma, (H)Thymoma, (I)Thyroid carcinoma, (J)Uterine corpus endometrial carcinoma. 

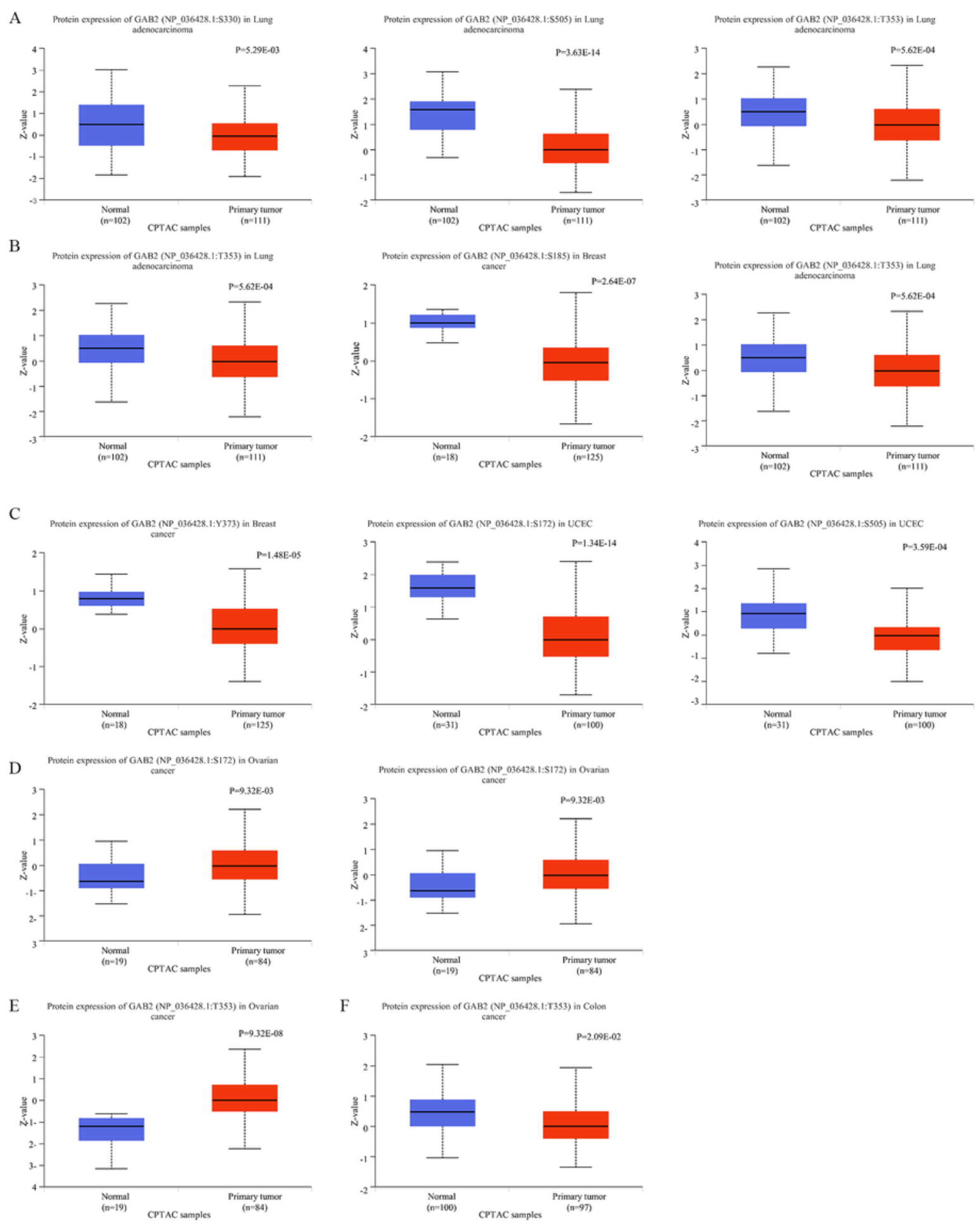

\section{Figure 3}

Phosphorylation analysis of GAB2 protein in different tumors. Based on the CPTAC dataset, we analyzed the expression level of GAB2 phosphoprotein between normal tissue and tumor tissue. We supply the box plots for different cancers, including Lung adenocarcinoma (A), breast cancer(B), UCEC(C), Ovarian cancer(D), Colon cancer(E), clear cancer RCC(F). 


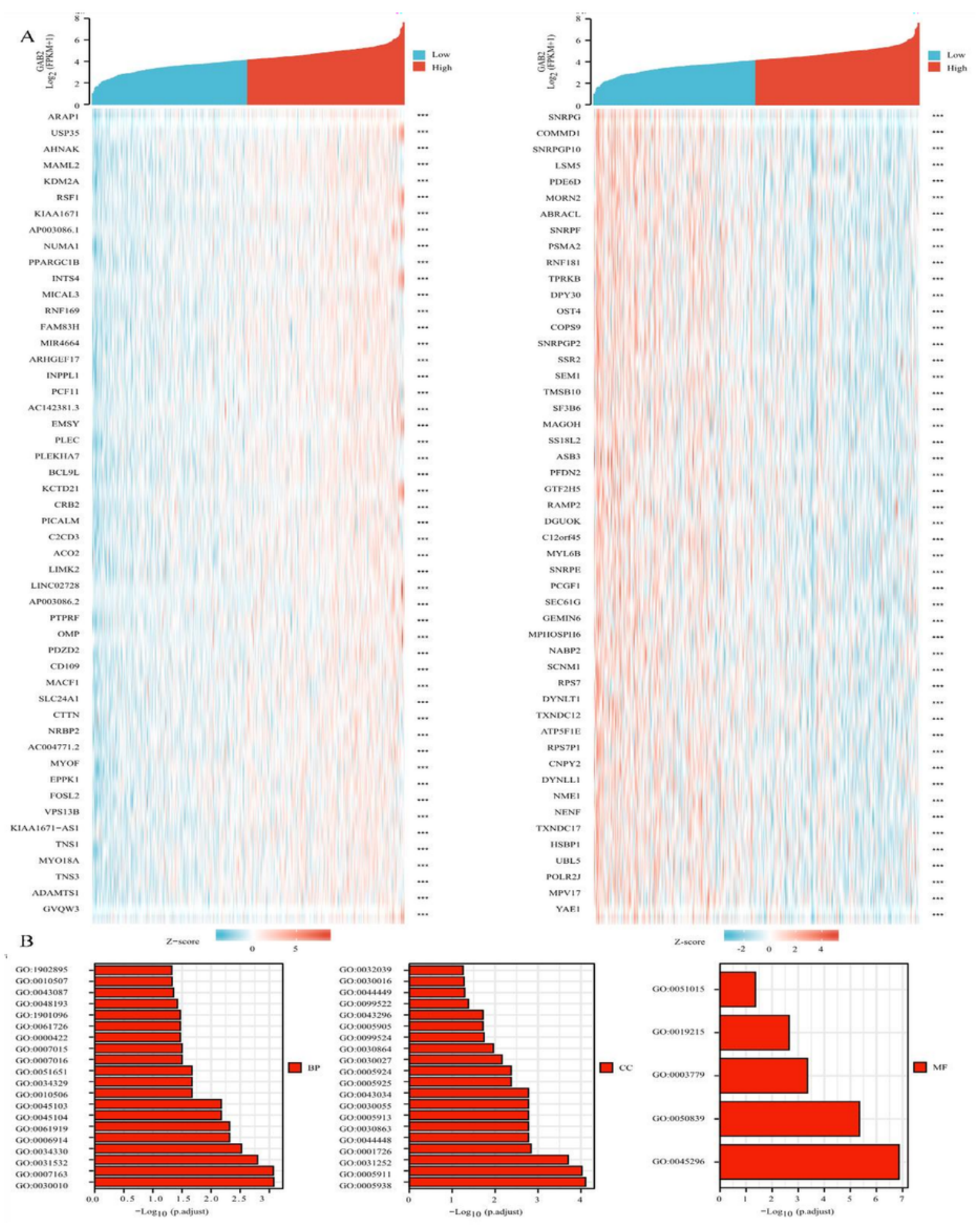

Figure 4

Expression of GAB2-related genes. (A)Top 50 genes most positively and negatively associated with GAB2 are shown in a heatmap. (B) Significant Gene Ontology terms of the top 300 genes most positively associated with GAB2, including biological processes (BP), molecular function (MF), and cell component (CC). 

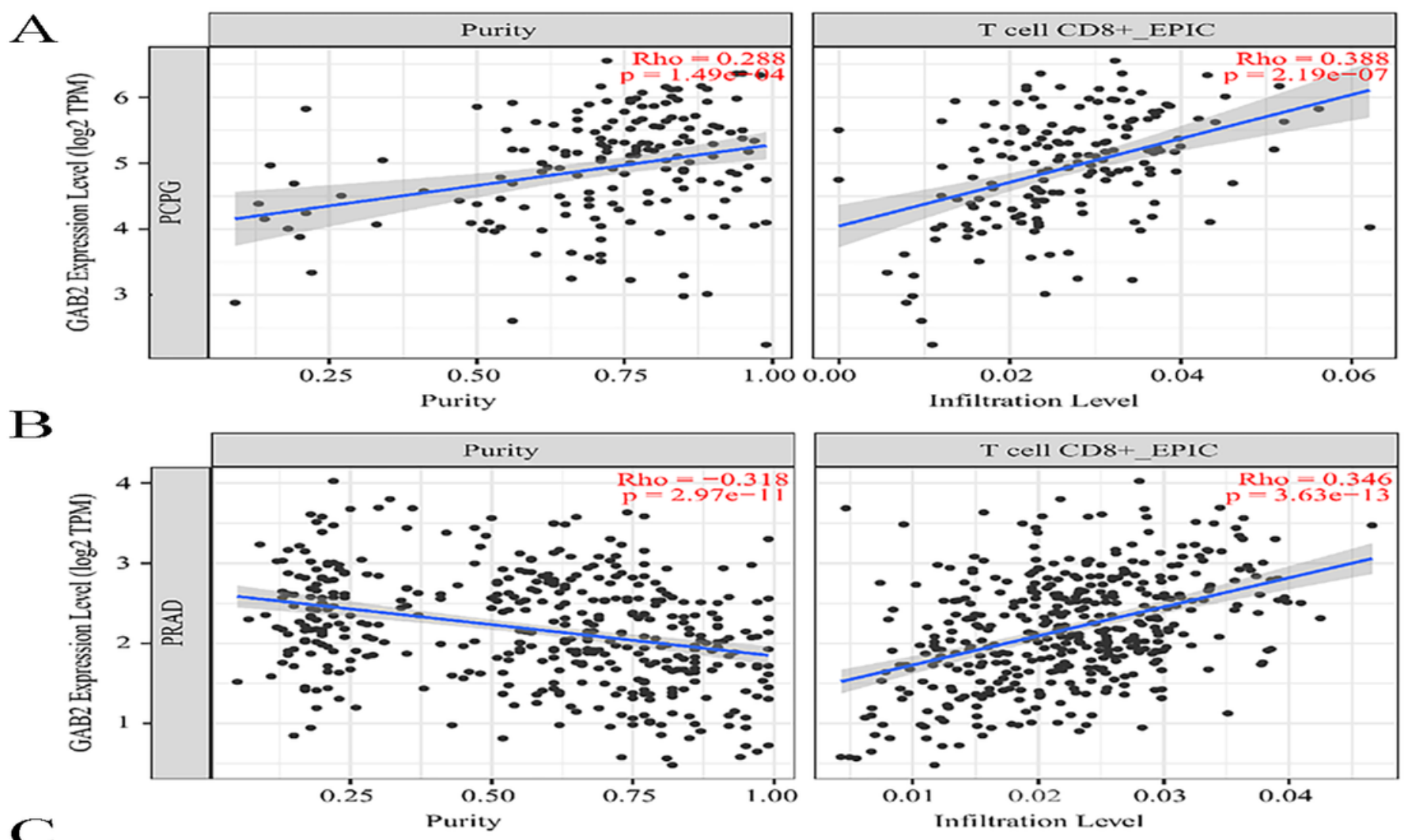

C
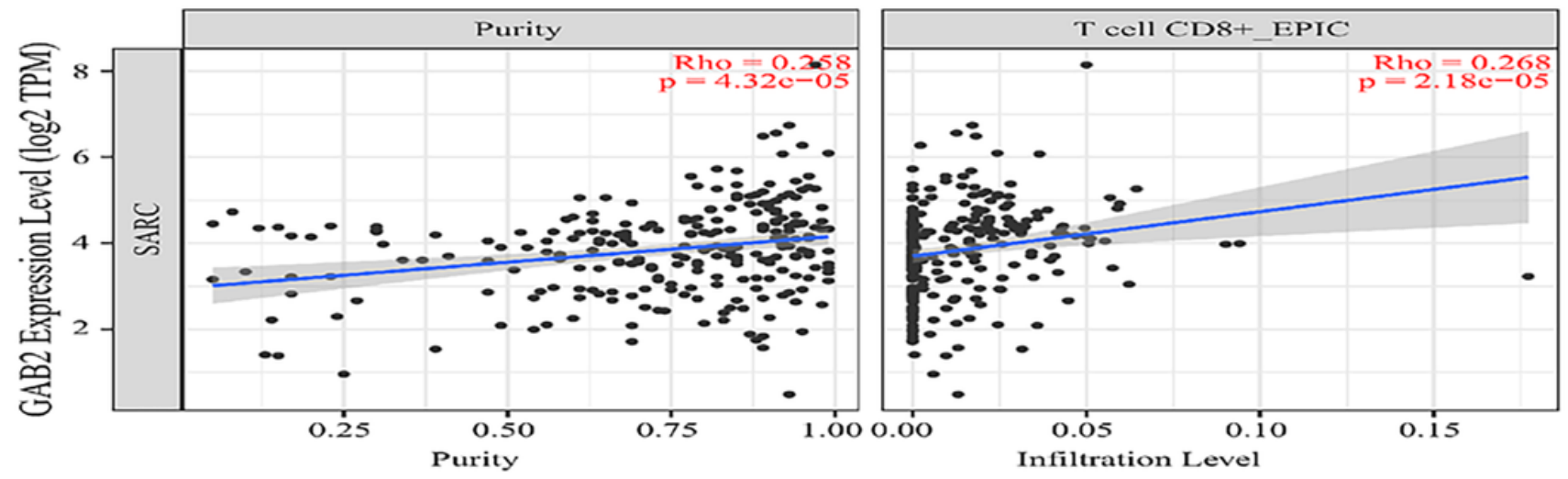

Figure 5

Correlation between GAB2 expression and CD8+T cell infiltration. Correlation between GAB2 expression and $C D 8+T$ cell infiltration levels using EPIC in different tumors, (A)PCPG, (B)PRAD, (C)SARC. 